\title{
Review Article \\ The Emerging Role of miRNAs and Their Clinical Implication in Biliary Tract Cancer
}

\author{
Nina Nayara Ferreira Martins, ${ }^{1}$ Kelly Cristina da Silva Oliveira, ${ }^{1}$ Amanda Braga Bona, ${ }^{2}$ \\ Marília de Arruda Cardoso Smith, ${ }^{3}$ Geraldo Ishak, ${ }^{1}$ Paulo Pimentel Assumpção, ${ }^{1}$ \\ Rommel Rodríguez Burbano, ${ }^{2}$ and Danielle Queiroz Calcagno ${ }^{1}$
}

\author{
${ }^{1}$ Núcleo de Pesquisas em Oncologia, Universidade Federal do Pará, Belém, PA, Brazil \\ ${ }^{2}$ Laboratório de Citogenética Humana, Instituto de Ciências Biológicas, Universidade Federal do Pará, Belém, PA, Brazil \\ ${ }^{3}$ Universidade Federal de São Paulo, São Paulo, SP, Brazil
}

Correspondence should be addressed to Danielle Queiroz Calcagno; danicalcagno@gmail.com

Received 22 July 2016; Revised 7 October 2016; Accepted 4 December 2016

Academic Editor: Mario Scartozzi

Copyright (C) 2016 Nina Nayara Ferreira Martins et al. This is an open access article distributed under the Creative Commons Attribution License, which permits unrestricted use, distribution, and reproduction in any medium, provided the original work is properly cited.

\begin{abstract}
Biliary tract cancers are aggressive malignancies that include gallbladder cancer and tumors of intra- and extrahepatic ducts and have a poor prognosis. Surgical resection remains the main curative therapy. Nevertheless, numerous patients experience recurrence even after radical surgery. This scenario drives the research to identify biliary tract cancer biomarkers despite the limited progress that has been made. Recently, a large number of studies have demonstrated that deregulated expression of microRNAs is closely associated with cancer development and progression. In this review, we highlight the role and importance of microRNAs in biliary tract cancers with an emphasis on utilizing circulating microRNAs as potential biomarkers. Additionally, we report several singlenucleotide polymorphisms in microRNA genes that are associated with the susceptibility of biliary tract tumors.
\end{abstract}

\section{Background}

Despite their relatively rare incidence, biliary tract cancers (BTCs) are an aggressive tumor group with poor prognosis and are characterized by early lymph node and systemic metastases [1]. These tumors include gallbladder cancer (GBC) and cholangiocarcinoma (CCA), which is divided into intrahepatic cholangiocarcinoma (iCCA) and extrahepatic cholangiocarcinoma (eCCA). Currently, surgical resection remains the only curative treatment for BTCs, and neoadjuvant chemoradiotherapy is not a standard option for patients with these malignancies. Moreover, many cases present with recurrence even after radical surgery, and patients with recurrent or metastatic BTCs usually have a poor outcome [2]. Therefore, there is a need for additional investigations to determine potential biomarkers of BTCs for early diagnosis, determining patient prognosis and the development of targeted therapy.
Recent studies have described microRNAs (miRNAs) as potential biomarkers in different cancer types [3-6]. However, miRNA expression and their implications in the diagnosis of, prognosis of, and therapeutic applications towards BTCs remain elusive.

miRNAs are small noncoding RNAs (18-25 nucleotides) that play important roles in the regulation of a large number of essential biological functions that are critical to the development of different cancer types, including cell proliferation, differentiation, apoptosis, migration, and invasion [7].

miRNA biogenesis initiates in the nucleus, where miRNA genes are usually transcribed by RNA polymerase II, resulting in a primary transcript of miRNA (pri-miRNAs) [8]. During the initial processing of pri-miRNAs, the DroshaDGCR8 complex cleaves the pri-miRNA, releasing a hairpin structure named pre-miRNA ( 70 nucleotides). Pre-miRNAs are transferred to the cytoplasm and converted into an miRNA duplex by Exportin-5 and the Dicer-TRBP complex, 
respectively. Then, a helicase separates the double-stranded miRNA to produce one stable single-stranded miRNA, while the other strand is processed for autolytic degradation. The stable mature miRNA strand is loaded into the RNAinduced silencing complex (RISC) to mechanistically target the $3^{\prime}$ untranslated regions ( $3^{\prime}$ UTRs) of protein coding mRNAs, thereby acting as posttranscriptional regulators by two mechanisms: mRNA degradation (when the sequences are perfect complements) and inhibition of translational initiation (when there is partial complementarily) [9]. Thus, miRNAs act as negative regulators of posttranscriptional gene expression of target mRNAs.

It has been well established that miRNAs could regulate approximately $60 \%$ of human genes, including many oncogenes and tumor suppressor genes; this phenomenon strengthens the importance of these noncoding RNAs as relevant regulators in cancer [10].

In this review, we focus on the roles and importance of miRNAs in BTCs and highlight the potential of circulating miRNAs as diagnostic and prognostic biomarkers. Therefore, we reported several single-nucleotide polymorphisms (SNPs) in miRNA genes associated with BTC susceptibility.

\section{Roles and Clinical Significance of miRNAs in BTCs}

A large number of deregulated miRNAs have been categorized as oncomiRs (oncogene miRNAs) and/or tsmiRs (tumor suppressor miRNAs) in cancer depending on the effect of the target mRNA.

In BTCs, several studies on miRNA expression have identified many upregulated oncomiRs and downregulated tsmiRs as well as their potential targets (Table 1).

One of the best-described miRNAs in BTCs is hsa-miR21 , which is usually identified as an oncomiR since its overexpression has been associated with invasion and metastasis [11-19, 21-25, 27, 30, 41, 47-50]. Liu et al. [13] observed that overexpression of hsa-miR-21 significantly promotes cell migration, invasion, and xenograft growth after transfection of hsa-miR-21 into CCA cell lines (QBC939 and RBE). Moreover, these authors showed decreased E-cadherin expression and increased $\mathrm{N}$-cadherin and vimentin expression after hsa-miR-21 overexpression. Thus, hsa-miR-21 could induce the epithelial-mesenchymal transition (EMT) in CCA. In this process, epithelial cells lose their cell polarity and cell adhesion-probably due to the decrease of E-cadherin expression-which allows cells to migrate and invade surrounding tissues; this the loss of E-cadherin expression plays a key role in tumor invasion and metastasis.

Similarly, aberrant expression of miRNAs also induces EMT and enhances the metastatic potential of GBC cells [24, 51]. Bao et al. [51] reported that hsa-miR-101 overexpression inhibits the proliferation, migration, and invasion of GBC cells, induces the increased expression of E-cadherin and $\beta$-catenin, and causes decreased expression of vimentin. Furthermore, these authors observed that hsa-miR-101 downregulation was correlated with tumor size, invasion, lymph node metastasis, TNM stage, and poor survival in GBC patients. These results indicate that hsa-miR-101 plays a tsmiR role and attenuates EMT and metastasis in GBC.

Accumulating evidence has indicated that hsa-miR-146b$5 p$ presents critical tumor suppressor properties $[52,53]$. Its expression was significantly downregulated in GBC tissue compared with adjacent nonneoplastic tissues. In addition, the overexpression of hsa-miR-146b-5p in the SGC-996 GBC cell line inhibited cell growth by enhancing apoptosis and arresting the cells at G1 phase. However, the enforced expression of EGFR, a cell surface protein that binds to epidermal growth factor (which inducing cell proliferation), reversed the ability of hsa-miR-146b-5p to inhibit proliferation. Moreover, hsa-miR-146b-5p expression levels were significantly correlated with tumor size and cancer progression [46].

Recent studies have described hsa-miR-135a-5p as having a tsmiR role [54-56]. In GBC, Zhou et al. [44] found that hsa-miR-135a-5p levels were significantly downregulated in tumors compared to adjacent nontumor gallbladder tissues and were correlated with neoplasms of histological grades III and IV. Additionally, this study identified VLDLR as a direct and functional target gene of hsa-miR-135a-5p in GBC tissues. Furthermore, the transfection with a hsa-miR-135a$5 \mathrm{p}$ mimetic inhibited the proliferative and colony-forming abilities of GBC cells by arresting the cells in G1/S phase. These data suggest that hsa-miR-135a-5p may inhibit the proliferation of GBC cells.

\section{Circulating miRNAs as Potential BTCs Biomarkers}

Several studies have reported that detectable miRNAs in bodily fluids (e.g., plasma, serum, urine, and saliva) are more stable in comparison with other circulating nucleic acids [57]. Therefore, circulating miRNAs may be noninvasive and specific diagnostic and/or prognostic molecular biomarkers for human diseases, including cancer $[4,7,58,59]$. In BTCs, many circulating miRNAs seem to be reproducible and reliable potential biomarkers as well as possible therapeutic targets [60]. Table 2 summarizes the circulating miRNAs with potential diagnostic, prognostic, and predictive biomarker applications in BTCs.

In CCA patients, Cheng et al. [64] observed different expression levels of circulating hsa-miR-106a not only between CCAs and healthy controls but also among CCAs and benign bile duct diseases (e.g., primary bile duct stone and congenital biliary duct cysts). Furthermore, they identified decreased hsa-miR-106a levels in patients with lymph node metastasis compared with those without metastasis, indicating the possible role of hsa-miR-106a in the occurrence of lymph node metastasis.

Interestingly, Voigtländer et al. [65] found a distinct circulating miRNA profile in the bile and serum samples from CCA patients and patients with primary sclerosing cholangitis (PSC), a noncancerous disease. Furthermore, bile samples from patients with concomitant PSC and CCA (PSC/CCA) were also included in this study. Their results showed higher expression levels of hsa-miR-126, hsa-miR-26a, hsa-miR-30b, hsa-miR-122, and hsa-miR-1281 in PSC patients than those 
TABLE 1: Deregulated miRNAs in BTCs.

\begin{tabular}{|c|c|c|c|c|c|}
\hline miRNA & Tumor & Expression & Target & Roles in BTCs & Reference \\
\hline \multirow{7}{*}{ hsa-miR-21 } & \multirow{7}{*}{ CCA } & $\uparrow$ & PTEN & $\begin{array}{c}\text { Invasion } \\
\text { Migration } \\
\text { Chemoresistance }\end{array}$ & {$[11-14]$} \\
\hline & & $\downarrow$ & TPM1 & $\begin{array}{l}\text { DNA methylation } \\
\text { Histone deacetylation }\end{array}$ & {$[15]$} \\
\hline & & $\downarrow$ & $\begin{array}{c}15-P G D H \\
H P G D\end{array}$ & Inflammation & {$[16]$} \\
\hline & & $\uparrow$ & PDCD4 & $\begin{array}{c}\text { Lymph node metastasis } \\
\text { Migration }\end{array}$ & {$[17-20]$} \\
\hline & & $\uparrow$ & $R E C K$ & $\begin{array}{l}\text { Migration } \\
\text { Metastasis }\end{array}$ & {$[21,22]$} \\
\hline & & $\uparrow$ & TIMP3 & Apoptosis & {$[18]$} \\
\hline & & $\uparrow$ & - & $\begin{array}{c}\text { pTNM } \\
\text { Prognosis }\end{array}$ & {$[23]$} \\
\hline hsa-miR-20 $0^{\mathrm{a}}$ & GBC & $\uparrow$ & SMAD7 & $\begin{array}{l}\text { Invasion } \\
\text { Metastasis } \\
\text { Migration } \\
\text { Prognosis } \\
\end{array}$ & {$[24]$} \\
\hline \multirow{2}{*}{ hsa-miR-34a } & CCA & $\downarrow$ & $C-M Y C$ & Progression & {$[25]$} \\
\hline & GBC & $\downarrow$ & PNUTS & $\begin{array}{l}\text { Proliferation } \\
\text { Prognosis }\end{array}$ & {$[26]$} \\
\hline hsa-miR-335 & GBC & $\downarrow$ & BMI1 & $\begin{array}{c}\text { Invasion } \\
\text { Lymph node metastasis } \\
\text { pTNM } \\
\text { Prognosis }\end{array}$ & {$[27,28]$} \\
\hline hsa-miR-148a & CCA & $\downarrow$ & DNMT1 & Prognosis & [29] \\
\hline hsa-miR-31 & CCA & $\uparrow$ & RASA1 & $\begin{array}{c}\text { Apoptosis } \\
\text { Proliferation }\end{array}$ & {$[30]$} \\
\hline hsa-miR-200b/c & CCA & $\downarrow$ & SUZ12 & $\begin{array}{l}\text { Chemoresistance } \\
\text { Invasion } \\
\text { Migration } \\
\end{array}$ & {$[28]$} \\
\hline hsa-miR-210 & CCA & $\uparrow$ & $M N T$ & Progression & {$[25]$} \\
\hline Let-7a & CCA & $\downarrow$ & $R A S$ & Progression & [21] \\
\hline hsa-miR-370 & CCA & $\downarrow$ & MAP3K8 & $\begin{array}{l}\text { Inflammation } \\
\text { pTNM }\end{array}$ & {$[31]$} \\
\hline hsa-miR-29b & CCA & $\downarrow$ & $C-M Y C$ & Apoptosis & {$[32]$} \\
\hline hsa-miR-101 & CCA & $\downarrow$ & $\begin{array}{l}\text { VEGF } \\
C O X-2\end{array}$ & Angiogenesis & {$[33]$} \\
\hline hsa-miR-200b/c & CCA & $\downarrow$ & ROCK2 & Migration & {$[28]$} \\
\hline hsa-miR-138 & $\mathrm{CCA}$ & $\downarrow$ & RHOC & Migration & {$[34]$} \\
\hline hsa-miR-376c & CCA & $\downarrow$ & GRB2 & Migration & [35] \\
\hline hsa-miR-124 & CCA & $\downarrow$ & SMYD3 & Migration & {$[36]$} \\
\hline hsa-miR-204 & CCA & $\downarrow$ & $S L U G$ & Migration & {$[37]$} \\
\hline hsa-miR-214 & CCA & $\downarrow$ & TWIST & Migration & {$[38]$} \\
\hline hsa-miR-200c & $\mathrm{CCA}$ & $\downarrow$ & NCAM1 & Migration & {$[39]$} \\
\hline hsa-miR-200b & $\mathrm{CCA}$ & $\uparrow$ & PTPN12 & Chemoresistance & {$[14]$} \\
\hline hsa-miR-29b & CCA & $\downarrow$ & $\begin{array}{l}P I K 3 R 1 \\
M M P 2\end{array}$ & Chemoresistance & \\
\hline hsa-miR-205 & CCA & $\downarrow$ & - & Chemoresistance & {$[40]$} \\
\hline hsa-miR-221 & $\mathrm{CCA}$ & $\downarrow$ & PIK3R1 & Chemoresistance & \\
\hline hsa-miR-182 & GBC & $\uparrow$ & $C A D M 1$ & $\begin{array}{c}\text { Invasion } \\
\text { Migration } \\
\text { Metastasis } \\
\end{array}$ & {$[27]$} \\
\hline
\end{tabular}


TABLE 1: Continued.

\begin{tabular}{|c|c|c|c|c|c|}
\hline miRNA & Tumor & Expression & Target & Roles in BTCs & Reference \\
\hline hsa-miR-155 & GBC & $\uparrow$ & SMAD7 & $\begin{array}{c}\text { Invasion } \\
\text { Lymph node metastasis } \\
\text { Proliferation } \\
\text { Prognosis }\end{array}$ & {$[41]$} \\
\hline hsa-miR-130a & GBC & $\downarrow$ & HOTAIR & $\begin{array}{c}\text { Invasion } \\
\text { Proliferation }\end{array}$ & {$[42]$} \\
\hline hsa-miR-26a & GBC & $\downarrow$ & HMGA2 & $\begin{array}{c}\text { pTNM } \\
\text { Proliferation }\end{array}$ & {$[43]$} \\
\hline hsa-miR-135a-5p & GBC & $\downarrow$ & $V L D L R$ & $\begin{array}{c}\text { pTNM } \\
\text { Proliferation }\end{array}$ & {$[44]$} \\
\hline hsa-miR-218-5p & GBC & $\downarrow$ & BMI1 & $\begin{array}{c}\text { Invasion } \\
\text { Migration } \\
\text { Proliferation } \\
\end{array}$ & {$[45]$} \\
\hline hsa-miR-146-5p & GBC & $\downarrow$ & $E G F R$ & $\begin{array}{c}\text { Apoptosis } \\
\text { pTNM } \\
\text { Proliferation }\end{array}$ & {$[46]$} \\
\hline hsa-miR-1 & GBC & $\downarrow$ & $\begin{array}{c}V E G F-A \\
A X L\end{array}$ & $\begin{array}{c}\text { Apoptosis } \\
\text { Proliferation }\end{array}$ & \\
\hline hsa-miR-145 & GBC & & $A X L$ & & {$[47]$} \\
\hline hsa-miR-143 & $\mathrm{GBC}$ & $\downarrow$ & $A X L$ & Lymph node metastasis & \\
\hline $\begin{array}{l}\text { hsa-miR-122 } \\
\text { hsa-miR-187 }\end{array}$ & GBC & $\uparrow$ & & pTNM stage & \\
\hline
\end{tabular}

in CCA patients. However, bile samples showed hsa-miR640, hsa-miR-1537, and hsa-miR-3189 downregulation, as well as hsa-miR-412 upregulation in PSC and PSC/CCA patients. These results demonstrated that PSC and CCA patients have distinct miRNA profiles in their bile and serum, which could be used to discriminate these diseases.

A small number of studies have described circulating miRNAs in patients with GBC. Kishimoto et al. [62] demonstrated an increase in the hsa-miR-21 expression levels in plasma from GBC patients before curative resection when compared with postsurgical patients and healthy volunteers. These findings suggest that hsa-miR-21 plasma levels were significantly affected by cancer occurrence and might have the potential to be a diagnostic biomarker for GBC patients.

Recently, Li and $\mathrm{Pu}$ [47] described significantly deregulated miRNAs in the peripheral blood samples of GBC patients compared with healthy volunteers. The expression levels of hsa-miR-187, hsa-miR-192, and hsa-miR-202 were upregulated while hsa-miR-143 was downregulated. These results were associated with lymph node metastasis, inflammation, immune reaction, and poor prognosis and could be translated to clinical practice as biomarkers for the early diagnosis, prognosis, and predictive response in patients with GBC.

Although most studies involving circulating miRNAs utilize real-time PCR for detection, Kojima et al. [68] used a highly sensitive microarray denoted as "3D Gene" that was capable of simultaneously analyzing more than 2,500 miRNAs in serum samples from patients with pancreatobiliary cancers. These authors found several significantly dysregulated miRNAs, including 30 upregulated miRNAs and 36 downregulated miRNAs in BTCs. However, none of these miRNAs could be used as single biomarker for this type of cancer. The best results were achieved with a panel of eight miRNAs (hsa-miR-6075, hsa-miR-4294, hsa-miR6880-5p, hsa-miR-6799-5p, hsa-miR-125a-3p, hsa-miR-4530, hsa-miR-6836-3p, and hsa-miR-4476).

\section{4. miRNA Single-Nucleotide Polymorphisms in BTCs}

In general, aberrations in miRNA expression result from either epigenetic modifications or genomic changes, which include chromosomal rearrangements, mutations, or SNPs [69].

Several SNPs in miRNAs can lead to distinctions in the miRNA expression levels, which can modulate miRNA-target gene expression and, subsequently, affect cancer susceptibility $[4,70]$. However, few studies have been performed to identify SNPs in miRNAs in BTCs patients until now.

The SNPs hsa-miR-27a rs895819, hsa-miR-570 rs4143815, and hsa-miR-181a rs12537 have been found to play important roles in many cancer types [71-78], and their contribution in BTCs has been explored. Gupta et al. [70] observed that the combination of hsa-miR-27a rs895819, hsa-miR570 rs4143815, and hsa-miR-181a rs12537 was the best genegene interaction model for predicting the susceptibility and treatment response in GBC patients. Moreover, the SNPs hsamiR-27a rs895819 and hsa-miR-181a rs12537 were associated with treatment toxicity but had no influence on the survival outcomes of GBC patients with locally advanced and/or metastatic tumors. 
TABLE 2: Circulating miRNAs in patients with BTC as potential diagnostic, prognostic, and predictive biomarkers.

\begin{tabular}{|c|c|c|c|c|c|c|c|}
\hline miRNA & Expression & Samples & N samples & $\begin{array}{c}\text { Potential } \\
\text { biomarker }\end{array}$ & Method & Clinical implication & Reference \\
\hline hsa-miR-9 & $\uparrow$ & Bile & $\begin{array}{c}\text { BTCs (9) } \\
\text { HV (9) }\end{array}$ & $\begin{array}{l}\text { Diagnostic } \\
\text { Prognostic }\end{array}$ & RT-PCR & Metastasis & {$[61]$} \\
\hline hsa-miR-145 & $\uparrow$ & Bile & $\begin{array}{c}\text { BTCs (9) } \\
\text { HV (9) }\end{array}$ & Diagnostic & RT-PCR & - & {$[61]$} \\
\hline \multirow[t]{2}{*}{ hsa-miR-21 } & \multirow[t]{2}{*}{$\uparrow$} & Plasma & $\begin{array}{l}\text { BTCs (94) } \\
\mathrm{HV}(50) \\
\operatorname{BBD}(2)\end{array}$ & Diagnostic & qRT-PCR & Inflammatory reaction & {$[62]$} \\
\hline & & Peripheral blood & $\begin{array}{l}\text { GBC (40) } \\
\mathrm{HV}(40)\end{array}$ & Diagnostic & qRT-PCR & - & {$[47]$} \\
\hline hsa-miR-150 & $\uparrow$ & Plasma & iCCA (15) & Diagnostic & qRT-PCR & Tumor progression & {$[63]$} \\
\hline hsa-miR-106a & $\downarrow$ & Serum & $\begin{array}{l}\text { CCA (103) } \\
\text { HV (20) }\end{array}$ & Prognostic & qRT-PCR & Lymph node metastasis & {$[64]$} \\
\hline hsa-miR-126 & $\uparrow$ & Serum & $\begin{array}{c}\text { PSC (40) } \\
\text { CCA (31) } \\
\text { HV (12) }\end{array}$ & Diagnostic & RT-PCR & - & {$[65]$} \\
\hline hsa-miR-26a & $\uparrow$ & Serum & $\begin{array}{l}\text { PSC (40) } \\
\text { CCA (31) } \\
\text { HV (12) }\end{array}$ & Diagnostic & RT-PCR & - & {$[65]$} \\
\hline hsa-miR-30b & $\uparrow$ & Serum & $\begin{array}{l}\text { PSC (40) } \\
\text { CC (31) } \\
\text { HV (12) }\end{array}$ & Diagnostic & RT-PCR & - & {$[65]$} \\
\hline hsa- miR-122 & $\uparrow$ & Serum & $\begin{array}{l}\text { PSC (40) } \\
\text { CC (31) } \\
\text { HV (12) } \\
\end{array}$ & Diagnostic & RT-PCR & - & {$[65]$} \\
\hline hsa-miR-1281 & $\uparrow$ & Serum & $\begin{array}{l}\text { PSC (40) } \\
\text { CC (31) } \\
\text { HV (12) }\end{array}$ & Diagnostic & RT-PCR & - & {$[65]$} \\
\hline hsa-miR -187 & $\uparrow$ & Peripheral blood & $\begin{array}{l}\text { GBC (40) } \\
\text { HV (40) }\end{array}$ & $\begin{array}{l}\text { Diagnostic } \\
\text { Prognostic } \\
\text { Predictive } \\
\end{array}$ & qRT-PCR & $\begin{array}{l}\text { Lymph node metastasis } \\
\text { Poor prognosis }\end{array}$ & {$[47]$} \\
\hline \multirow[t]{2}{*}{ hsa-miR-192 } & \multirow[t]{2}{*}{$\uparrow$} & Peripheral blood & $\begin{array}{l}\text { GBC (40) } \\
\mathrm{HV}(40)\end{array}$ & $\begin{array}{l}\text { Diagnostic } \\
\text { Prognostic } \\
\text { Predictive }\end{array}$ & qRT-PCR & $\begin{array}{l}\text { Inflammatory reaction } \\
\text { Immune reaction } \\
\text { Lymph node metastasis }\end{array}$ & {$[47]$} \\
\hline & & Serum & $\begin{array}{l}\text { iCCA (11) } \\
\mathrm{HV}(09)\end{array}$ & $\begin{array}{l}\text { Diagnostic } \\
\text { Prognostic }\end{array}$ & $\begin{array}{c}\text { miRNA } \\
\text { RT-PCR array }\end{array}$ & $\begin{array}{l}\text { Lymph node metastasis } \\
\text { Poor prognosis }\end{array}$ & {$[66]$} \\
\hline hsa-miR-194 & $\uparrow$ & Serum & $\begin{array}{l}\text { CCA (70) } \\
\mathrm{HV}(70)\end{array}$ & Diagnostic & qRT-PCR & Tumor progression & {$[58]$} \\
\hline hsa-miR -202 & $\uparrow$ & Peripheral blood & $\begin{array}{l}\text { GBC (40) } \\
\mathrm{HV}(40)\end{array}$ & $\begin{array}{l}\text { Diagnostic } \\
\text { Prognostic } \\
\text { Predictive } \\
\end{array}$ & qRT-PCR & Lymph node metastasis & {$[47]$} \\
\hline hsa-let- 7a & $\downarrow$ & Peripheral blood & $\begin{array}{l}\text { GBC (40) } \\
\mathrm{HV}(40)\end{array}$ & Diagnostic & qRT-PCR & - & {$[47]$} \\
\hline hsa-miR -143 & $\downarrow$ & Peripheral blood & $\begin{array}{l}\text { GBC (40) } \\
\mathrm{HV}(40)\end{array}$ & $\begin{array}{l}\text { Diagnostic } \\
\text { Prognostic } \\
\text { Predictive } \\
\end{array}$ & qRT-PCR & $\begin{array}{c}\text { Inflammatory and } \\
\text { immune reaction } \\
\text { Lymph node metastasis }\end{array}$ & {$[47]$} \\
\hline hsa-miR-335 & $\downarrow$ & Peripheral blood & $\begin{array}{l}\text { GBC (40) } \\
\text { HV (40) }\end{array}$ & Diagnostic & qRT-PCR & - & {$[47]$} \\
\hline hsa-miR-1307-3p & $\downarrow$ & Plasma & $\begin{array}{l}\text { iCCA (13) } \\
\text { HV (5) }\end{array}$ & Diagnostic & qRT-PCR & - & {$[67]$} \\
\hline hsa-miR-1275 & $\uparrow$ & Plasma & $\begin{array}{c}\text { iCCA (13) } \\
\text { HV (5) }\end{array}$ & Diagnostic & qRT-PCR & - & {$[67]$} \\
\hline
\end{tabular}


TABLE 2: Continued.

\begin{tabular}{|c|c|c|c|c|c|c|c|}
\hline miRNA & Expression & Samples & $\mathrm{N}$ samples & $\begin{array}{c}\text { Potential } \\
\text { biomarker }\end{array}$ & Method & Clinical implication & Reference \\
\hline hsa- miR-320b & $\uparrow$ & Plasma & $\begin{array}{c}\text { iCCA (13) } \\
\text { HVs (5) }\end{array}$ & Diagnostic & qRT-PCR & - & {$[67]$} \\
\hline hsa-miR-874 & $\uparrow$ & Plasma & $\begin{array}{c}\text { iCCA (13) } \\
\text { HVs (5) }\end{array}$ & Diagnostic & qRT-PCR & - & {$[67]$} \\
\hline \multirow{2}{*}{ hsa-miR-483-5p } & \multirow{2}{*}{$\uparrow$} & Plasma & $\begin{array}{c}\text { iCCA (13) } \\
\text { HV (5) }\end{array}$ & Diagnostic & qRT-PCR & - & {$[67]$} \\
\hline & & Serum & $\begin{array}{c}\text { CCA (70) } \\
\text { HV (70) }\end{array}$ & Diagnostic & qRT-PCR & Tumor progression & {$[58]$} \\
\hline hsa-miR-885-5p & $\uparrow$ & Plasma & $\begin{array}{c}\text { iCCA (13) } \\
\text { HV (5) }\end{array}$ & Diagnostic & qRT-PCR & - & {$[67]$} \\
\hline hsa-miR-92b-3p & $\uparrow$ & Plasma & $\begin{array}{c}\text { iCCA (13) } \\
\text { HV (5) }\end{array}$ & Diagnostic & qRT-PCR & - & {$[67]$} \\
\hline hsa-miR-505-3p & $\uparrow$ & Plasma & $\begin{array}{c}\text { iCCA (13) } \\
\text { HV (5) }\end{array}$ & Diagnostic & qRT-PCR & - & {$[67]$} \\
\hline hsa-miR-6836-3p & $\uparrow$ & Serum & $\begin{array}{c}\text { BTCs (98) } \\
\text { HV (150) }\end{array}$ & Diagnostic & 3D-Gene & - & {$[68]$} \\
\hline hsa-miR-6075 & $\uparrow$ & Serum & $\begin{array}{c}\text { BTCs (98) } \\
\text { HV (150) }\end{array}$ & Diagnostic & 3D-Gene & - & {$[68]$} \\
\hline hsa- miR-4634 & $\uparrow$ & Serum & $\begin{array}{c}\text { BTCs (98) } \\
\text { HV (150) }\end{array}$ & Diagnostic & 3D-Gene & - & {$[68]$} \\
\hline hsa-miR-4294 & $\downarrow$ & Serum & $\begin{array}{c}\text { BTCs (98) } \\
\text { HV (150) }\end{array}$ & Diagnostic & 3D-Gene & - & {$[68]$} \\
\hline hsa-miR-6880-5p & $\downarrow$ & Serum & $\begin{array}{c}\text { BTCs (98) } \\
\text { HV (150) }\end{array}$ & Diagnostic & 3D-Gene & - & {$[68]$} \\
\hline hsa-miR-6799-5p & $\downarrow$ & Serum & $\begin{array}{c}\text { BTCs (98) } \\
\text { HV (150) }\end{array}$ & Diagnostic & 3D-Gene & - & {$[68]$} \\
\hline hsa-miR-125a-3p, & $\downarrow$ & Serum & $\begin{array}{c}\text { BTCs (98) } \\
\text { HV (150) }\end{array}$ & Diagnostic & 3D-Gene & Tumor progression & {$[68]$} \\
\hline hsa-miR-4530 & $\downarrow$ & Serum & $\begin{array}{c}\text { BTCs (98) } \\
\text { HV (150) }\end{array}$ & Diagnostic & 3D-Gene & - & {$[68]$} \\
\hline hsa-miR-7114-5p & $\downarrow$ & Serum & $\begin{array}{c}\text { BTCs (98) } \\
\text { HV (150) }\end{array}$ & Diagnostic & 3D-Gene & - & {$[68]$} \\
\hline hsa-miR-4476 & $\downarrow$ & Serum & $\begin{array}{c}\text { BTCs (98) } \\
\text { HV (150) }\end{array}$ & Diagnostic & 3D-Gene & - & {$[68]$} \\
\hline
\end{tabular}

BBD: benign biliary disorders; BTCs: biliary tract cancers; CCA: cholangiocarcinoma; iCCA: intrahepatic cholangiocarcinoma; GBC: gallbladder cancer; HV: heath volunteers; PSC: primary sclerosing cholangitis.

SNPs in pri-miRNAs and pre-miRNAs could also affect miRNA processing, miRNA expression, and cancer susceptibility. Srivastava et al. [79] reported genetic polymorphisms in pre-mir-196a2 rs11614913 (C>T), pre-hsa-mir-196a rs11614913, and pre-hsa-mir-499 rs3746444 (T>C) that were associated with an increased overall risk of developing GBC development. In CCA, Mihalache et al. [80] investigated the G/C variant in pre-hsa-miR-146a rs2910164 and found no significant relationship between genetic susceptibility and CCA.

Additional studies addressing the identification of miRNA SNPs could be useful to assess the individual susceptibility of BTCs and improve our understanding of their potential contribution to the disease as well as aid in the development of potential clinical applications.

\section{Conclusion}

miRNAs are profoundly involved in tumor onset and progression [81-84]. However, the implications of miRNA for the diagnosis, prognosis, and therapeutic options for patients with BTCs remain unsatisfactory. This review highlighted some miRNAs that are dysregulated in BTCs, their targets, and the possible clinical implications. A better understanding of the therapeutic applications of miRNAs could lead to future clinical trials involving the inhibition of oncomiRs or the promotion of expression of tsmiRs as new approaches against diverse cancer types, including aggressive BTCs.

Here, we also reported several circulating miRNAs as possible diagnostic, prognostic, and/or predictive biomarkers 
in BTCs. Circulating miRNAs could be promising potential biomarkers for cancers because detectable miRNAs in the bodily fluids are stable and can be measured using noninvasive methods [57]. BTCs are usually asymptomatic; therefore, the use of miRNAs as early diagnostic biomarkers could be a useful tool to improve the long-term survival of BTC patients. However, more studies with clinical outcomes are needed to identify which miRNAs could serve as either a potential therapeutic target or diagnostic and prognostic biomarkers of BTCs.

Moreover, several SNPs in miRNAs can affect the expression of target genes, leading to a cellular disorder and, consequently, tumorigenesis $[4,70]$. However, few studies have been performed to identify SNPs in the miRNAs expressed by BTC patients until now; this review emphasizes the need to expand the knowledge in this field of study.

\section{Competing Interests}

The authors declare no conflict of interests for this article.

\section{Authors' Contributions}

Danielle Queiroz Calcagno conceived the review design; Nina Nayara Ferreira Martins, Kelly Cristina da Silva Oliveira, and Danielle Queiroz Calcagno collected the data; Nina Nayara Ferreira Martins, Kelly Cristina da Silva Oliveira, Amanda Braga Bona, and Danielle Queiroz Calcagno wrote the paper; Marília de Arruda Cardoso Smith and Geraldo Ishak performed corrections and made suggestions; Paulo Pimentel Assumpção and Rommel Rodríguez Burbano critically revised the paper.

\section{Acknowledgments}

This study was supported by the Fundação de Amparo à Pesquisa do Estado de São Paulo (FAPESP, to Marília de Arruda Cardoso Smith), the Conselho Nacional de Desenvolvimento Científico e Tecnológico $(\mathrm{CNPq}$, to Marília de Arruda Cardoso Smith and Rommel Rodríguez Burbano), and the Coordenação de Aperfeiçoamento de Pessoal de Nível Superior (CAPES, to Nina Nayara Ferreira Martins and Kelly Cristina da Silva Oliveira).

\section{References}

[1] K. H. Yoo, N. K. D. Kim, W. I. Kwon et al., "Genomic alterations in biliary tract cancer using targeted sequencing," Translational Oncology, vol. 9, no. 3, pp. 173-178, 2016.

[2] K. K. Ciombor and L. W. Goff, "Advances in the management of biliary tract cancers," Clinical Advances in Hematology and Oncology, vol. 11, no. 1, pp. 28-34, 2013.

[3] C. O. Gigek, E. S. Chen, D. Q. Calcagno, F. Wisnieski, R. R. Burbano, and M. A. C. Smith, "Epigenetic mechanisms in gastric cancer," Epigenomics, vol. 4, no. 3, pp. 279-294, 2012.

[4] D. Q. Calcagno, M. D. A. Cardoso Smith, and R. R. Burbano, "Cancer type-specific epigenetic changes: gastric cancer," Methods in Molecular Biology, vol. 1238, pp. 79-101, 2015.
[5] A. Saumet and C.-H. Lecellier, "MicroRNAs and personalized medicine: evaluating their potential as cancer biomarkers," Advances in Experimental Medicine and Biology, vol. 888, pp. 5-15, 2015.

[6] D. Huo, W. M. Clayton, T. F. Yoshimatsu, J. Chen, and O. I. Olopade, "Identification of a circulating MicroRNA signature to distinguish recurrence in breast cancer patients," Oncotarget, 2016.

[7] G. Yang, L. Zhang, R. Li, and L. Wang, "The role of microRNAs in gallbladder cancer (Review)," Molecular and Clinical Oncology, vol. 5, no. 1, pp. 7-13, 2016.

[8] Y. Lee, M. Kim, J. Han et al., "MicroRNA genes are transcribed by RNA polymerase II," EMBO Journal, vol. 23, no. 20, pp. 40514060, 2004.

[9] S. Lin and R. I. Gregory, "MicroRNA biogenesis pathways in cancer," Nature Reviews Cancer, vol. 15, no. 6, pp. 321-333, 2015.

[10] C. Weber, "MicroRNAs: from basic mechanisms to clinical application in cardiovascular medicine," Arteriosclerosis, Thrombosis, and Vascular Biology, vol. 33, no. 2, pp. 168-169, 2013.

[11] J. Zhang, J. Jiao, S. Cermelli et al., "miR-21 inhibition reduces liver fibrosis and prevents tumor development by inducing apoptosis of CD $24^{+}$progenitor cells," Cancer Research, vol. 75, no. 9, pp. 1859-1867, 2015.

[12] Q. He, L. Cai, L. Shuai et al., "Ars2 is overexpressed in human cholangiocarcinomas and its depletion increases PTEN and PDCD 4 by decreasing microRNA-21," Molecular Carcinogenesis, vol. 52, no. 4, pp. 286-296, 2013.

[13] Z. Liu, Z.-Y. Jin, C.-H. Liu, F. Xie, X.-S. Lin, and Q. Huang, "MicroRNA-21 regulates biological behavior by inducing EMT in human cholangiocarcinoma," International Journal of Clinical and Experimental Pathology, vol. 8, no. 5, pp. 4684-4694, 2015.

[14] F. Meng, R. Henson, M. Lang et al., "Involvement of human micro-RNA in growth and response to chemotherapy in human cholangiocarcinoma cell lines," Gastroenterology, vol. 130, no. 7, pp. 2113-2129, 2006.

[15] W. Yang, X. Wang, W. Zheng, K. Li, H. Liu, and Y. Sun, "Genetic and epigenetic alterations are involved in the regulation of TPM1 in cholangiocarcinoma," International Journal of Oncology, vol. 42, no. 2, pp. 690-698, 2013.

[16] L. Lu, K. Byrnes, C. Han, Y. Wang, and T. Wu, "MiR-21 targets 15PGDH and promotes cholangiocarcinoma growth," Molecular Cancer Research, vol. 12, no. 6, pp. 890-900, 2014.

[17] P. Chusorn, N. Namwat, W. Loilome et al., "Overexpression of microRNA-21 regulating PDCD4 during tumorigenesis of liver fluke-associated cholangiocarcinoma contributes to tumor growth and metastasis," Tumor Biology, vol. 34, no. 3, pp. 15791588, 2013.

[18] F. M. Selaru, A. V. Olaru, T. Kan et al., "MicroRNA-21 is overexpressed in human cholangiocarcinoma and regulates programmed cell death 4 and tissue inhibitor of metalloproteinase 3," Hepatology, vol. 49, no. 5, pp. 1595-1601, 2009.

[19] C.-Z. Liu, W. Liu, Y. Zheng et al., "PTEN and PDCD4 are bona fide targets of microRNA-21 in human cholangiocarcinoma," Chinese Medical Sciences Journal, vol. 27, no. 2, pp. 65-72, 2012.

[20] J. He, Y. Yue, C. Dong, and S. Xiong, "MiR-21 confers resistance against CVB3-induced myocarditis by inhibiting PDCD4mediated apoptosis," Clinical and Investigative Medicine, vol. 36, no. 2, pp. E103-E111, 2013.

[21] N. Namwat, P. Chusorn, W. Loilome et al., "Expression profiles of oncomir miR-21 and tumor suppressor let-7a in the progression of opisthorchiasis-associated cholangiocarcinoma," Asian 
Pacific Journal of Cancer Prevention, vol. 13, supplement, pp. 6569, 2012.

[22] Q. Huang, L. Liu, C.-H. Liu et al., "MicroRNA-21 Regulates the invasion and metastasis in cholangiocarcinoma and may be a potential biomarker for cancer prognosis," Asian Pacific Journal of Cancer Prevention, vol. 14, no. 2, pp. 829-834, 2013.

[23] A. Karakatsanis, I. Papaconstantinou, M. Gazouli, A. Lyberopoulou, G. Polymeneas, and D. Voros, "Expression of microRNAs, miR-21, miR-31, miR-122, miR-145, miR-146a, miR-200c, miR-221, miR-222, and miR-223 in patients with hepatocellular carcinoma or intrahepatic cholangiocarcinoma and its prognostic significance," Molecular Carcinogenesis, vol. 52, no. 4, pp. 297-303, 2013.

[24] Y. Chang, J. Yang, G. Liu et al., "MiR-20a triggers metastasis of gallbladder carcinoma," Journal of Hepatology, vol. 59, no. 3, pp. 518-527, 2013.

[25] H. Yang, T. W. H. Li, J. Peng et al., "A mouse model of cholestasis-associated cholangiocarcinoma and transcription factors involved in progression," Gastroenterology, vol. 141, no. 1, pp. 378.e4-388.e4, 2011.

[26] K. Jin, Y. Xiang, J. Tang et al., "miR-34 is associated with poor prognosis of patients with gallbladder cancer through regulating telomere length in tumor stem cells," Tumor Biology, vol. 35, no. 2, pp. 1503-1510, 2014.

[27] Y. Qiu, X. Luo, T. Kan et al., “TGF- $\beta$ upregulates miR-182 expression to promote gallbladder cancer metastasis by targeting CADM1," Molecular BioSystems, vol. 10, no. 3, pp. 679-685, 2014.

[28] F. Peng, J. Jiang, Y. Yu et al., "Direct targeting of SUZ12/ROCK2 by $\mathrm{miR}-200 \mathrm{~b} / \mathrm{c}$ inhibits cholangiocarcinoma tumourigenesis and metastasis," British Journal of Cancer, vol. 109, no. 12, pp. 3092-3104, 2013.

[29] C. Braconi, N. Huang, and T. Patel, "Microrna-dependent regulation of DNA methyltransferase-1 and tumor suppressor gene expression by interleukin-6 in human malignant cholangiocytes," Hepatology, vol. 51, no. 3, pp. 881-890, 2010.

[30] C. Hu, F. Huang, G. Deng, W. Nie, W. Huang, and X. Zeng, "miR-31 promotes oncogenesis in intrahepatic cholangiocarcinoma cells via the direct suppression of RASA1," Experimental and Therapeutic Medicine, vol. 6, no. 5, pp. 1265-1270, 2013.

[31] F. Meng, H. Wehbe-Janek, R. Henson, H. Smith, and T. Patel, "Epigenetic regulation of microRNA-370 by interleukin- 6 in malignant human cholangiocytes," Oncogene, vol. 27, no. 3, pp. 378-386, 2008.

[32] J. L. Mott, S. Kurita, S. C. Cazanave, S. F. Bronk, N. W. Werneburg, and M. E. Fernandez-Zapico, "Transcriptional suppression of mir-29b-1/mir-29a promoter by c-Myc, hedgehog, and NFkappaB," Journal of Cellular Biochemistry, vol. 110, no. 5, pp. 1155-1164, 2010.

[33] J. Zhang, C. Han, H. Zhu, K. Song, and T. Wu, "MiR-101 inhibits cholangiocarcinoma angiogenesis through targeting vascular endothelial growth factor (VEGF)," American Journal of Pathology, vol. 182, no. 5, pp. 1629-1639, 2013.

[34] Q. Wang, H. Tang, S. Yin, and C. Dong, "Downregulation of microRNA-138 enhances the proliferation, migrationand invasion of cholangiocarcinoma cells through the upregulation of RhoC/p-ERK/MMP-2/MMP-9," Oncology Reports, vol. 29, no. 5, pp. 2046-2052, 2013.

[35] J. Iwaki, K. Kikuchi, Y. Mizuguchi et al., "MiR-376c downregulation accelerates EGF-dependent migration by targeting GRB2 in the HuCCT1 human intrahepatic cholangiocarcinoma cell line," PLoS ONE, vol. 8, no. 7, Article ID e69496, 2013.
[36] B. Zeng, Z. Li, R. Chen et al., "Epigenetic regulation of miR124 by Hepatitis $C$ Virus core protein promotes migration and invasion of intrahepatic cholangiocarcinoma cells by targeting SMYD3," FEBS Letters, vol. 586, no. 19, pp. 3271-3278, 2012.

[37] Y.-H. Qiu, Y.-P. Wei, N.-J. Shen et al., "MiR-204 Inhibits epithelial to mesenchymal transition by targeting slug in intrahepatic cholangiocarcinoma cells," Cellular Physiology and Biochemistry, vol. 32, no. 5, pp. 1331-1341, 2013.

[38] B. Li, Q. Han, Y. Zhu, Y. Yu, J. Wang, and X. Jiang, "Downregulation of miR-214 contributes to intrahepatic cholangiocarcinoma metastasis by targeting Twist," FEBS Journal, vol. 279, no. 13, pp. 2393-2398, 2012.

[39] N. Oishi, M. R. Kumar, S. Roessler et al., "Transcriptomic profiling reveals hepatic stem-like gene signatures and interplay of miR-200c and epithelial-mesenchymal transition in intrahepatic cholangiocarcinoma," Hepatology, vol. 56, no. 5, pp. 1792-1803, 2012.

[40] K. Okamoto, K. Miyoshi, and Y. Murawaki, "miR-29b, miR205 and miR-221 enhance chemosensitivity to gemcitabine in HuH28 human cholangiocarcinoma cells," PLOS ONE, vol. 8, no. 10, Article ID e77623, 2013.

[41] H. Kono, M. Nakamura, T. Ohtsuka et al., "High expression of microRNA-155 is associated with the aggressive malignant behavior of gallbladder carcinoma," Oncology reports, vol. 30, no. 1, pp. 17-24, 2013.

[42] M.-Z. Ma, C.-X. Li, Y. Zhang et al., "Long non-coding RNA HOTAIR, a c-Myc activated driver of malignancy, negatively regulates miRNA-130a in gallbladder cancer," Molecular Cancer, vol. 13, no. 1, article no. 156, 2014.

[43] H. Zhou, W. Guo, Y. Zhao et al., "MicroRNA-26a acts as a tumor suppressor inhibiting gallbladder cancer cell proliferation by directly targeting HMGA2," International Journal of Oncology, vol. 45, no. 6, pp. 2050-2058, 2014.

[44] H. Zhou, W. Guo, Y. Zhao et al., "MicroRNA-135a acts as a putative tumor suppressor by directly targeting very low density lipoprotein receptor in human gallbladder cancer," Cancer Science, vol. 105, no. 8, pp. 956-965, 2014.

[45] M.-Z. Ma, B.-F. Chu, Y. Zhang et al., "Long non-coding RNA CCAT1 promotes gallbladder cancer development via negative modulation of miRNA-218-5p," Cell Death and Disease, vol. 6, no. 1, Article ID e1583, 2015.

[46] J. Cai, L. Xu, Z. Cai, J. Wang, B. Zhou, and H. Hu, "MicroRNA146b-5p inhibits the growth of gallbladder carcinoma by targeting epidermal growth factor receptor," Molecular Medicine Reports, vol. 12, no. 1, pp. 1549-1555, 2015.

[47] G. Li and Y. Pu, "MicroRNA signatures in total peripheral blood of gallbladder cancer patients," Tumor Biology, vol. 36, no. 9, pp. 6985-6990, 2015.

[48] T. Kitamura, K. Connolly, L. Ruffino et al., "The therapeutic effect of histone deacetylase inhibitor PCI-24781 on gallbladder carcinoma in BK5.erbB2 mice," Journal of Hepatology, vol. 57, no. 1, pp. 84-91, 2012.

[49] L.-J. Wang, C.-C. He, X. Sui et al., "MiR-21 promotes intrahepatic cholangiocarcinoma proliferation and growth in vitro and in vivo by targeting PTPN14 and PTEN," Oncotarget, vol. 6, no. 8, pp. 5932-5946, 2015.

[50] S. Sekine, Y. Shimada, T. Nagata et al., "Role of aquaporin-5 in gallbladder carcinoma," European Surgical Research, vol. 51, no. 3-4, pp. 108-117, 2013.

[51] R. Bao, Y. Shu, Y. Hu et al., "miR-101 targeting ZFX suppresses tumor proliferation and metastasis by regulating the 
MAPK/Erk and smad pathways in gallbladder carcinoma," Oncotarget, 2016.

[52] P. Y. Wu, X. D. Zhang, J. Zhu, X. Y. Guo, and J. F. Wang, "Low expression of microRNA-146b-5p and microRNA-320d predicts poor outcome of large B-cell lymphoma treated with cyclophosphamide, doxorubicin, vincristine, and prednisone," Human Pathology, vol. 45, no. 8, pp. 1664-1673, 2014.

[53] C. Shen, H. Yang, H. Liu, X. Wang, Y. Zhang, and R. Xu, "Inhibitory effect and mechanisms of microRNA-146b-5p on the proliferation and metastatic potential of Caski human cervical cancer cells," Molecular Medicine Reports, vol. 11, no. 5, pp. 3955-3961, 2015.

[54] Z. Dang, W.-H. Xu, P. Lu et al., "MicroRNA-135a inhibits cell proliferation by targeting Bmil in pancreatic ductal adenocarcinoma," International Journal of Biological Sciences, vol. 10, no. 7, pp. 733-745, 2014.

[55] J.-Y. Shin, Y.-I. Kim, S.-J. Cho et al., "MicroRNA 135a suppresses lymph node metastasis through down-regulation of ROCK1 in early gastric cancer," PLoS ONE, vol. 9, no. 1, Article ID e85205, 2014.

[56] W. Tang, Y. Jiang, X. Mu, L. Xu, W. Cheng, and X. Wang, "MiR135a functions as a tumor suppressor in epithelial ovarian cancer and regulates HOXA10 expression," Cellular Signalling, vol. 26, no. 7, pp. 1420-1426, 2014.

[57] I. Igaz and P. Igaz, "Diagnostic relevance of microRNAs in other body fluids including urine, feces, and saliva," EXS, vol. 106, pp. 245-252, 2015.

[58] F. Bernuzzi, F. Marabita, A. Lleo et al., "Serum microRNAs as novel biomarkers for primary sclerosing cholangitis and cholangiocarcinoma," Clinical \& Experimental Immunology, vol. 185, no. 1, pp. 61-71, 2016.

[59] K. Piontek and F. M. Selaru, "MicroRNAs in the biology and diagnosis of cholangiocarcinoma," Seminars in Liver Disease, vol. 35, no. 1, pp. 55-62, 2015.

[60] P. Letelier, I. Riquelme, A. Hernández, N. Guzmán, J. Farías, and J. Roa, "Circulating MicroRNAs as biomarkers in biliary tract cancers," International Journal of Molecular Sciences, vol. 17, no. 5, p. 791, 2016.

[61] K. Shigehara, S. Yokomuro, O. Ishibashi et al., "Real-time PCRbased analysis of the human bile micrornaome identifies miR9 as a potential diagnostic biomarker for biliary tract cancer," PLoS ONE, vol. 6, no. 8, Article ID e23584, 2011.

[62] T. Kishimoto, H. Eguchi, H. Nagano et al., "Plasma miR-21 is a novel diagnostic biomarker for biliary tract cancer," Cancer Science, vol. 104, no. 12, pp. 1626-1631, 2013.

[63] S. Wang, J. Yin, T. Li et al., "Upregulated circulating miR-150 is associated with the risk of intrahepatic cholangiocarcinoma," Oncology Reports, vol. 33, no. 2, pp. 819-825, 2015.

[64] Q. Cheng, F. Feng, L. Zhu et al., "Circulating miR-106a is a novel prognostic and lymph node metastasis indicator for cholangiocarcinoma," Scientific Reports, vol. 5, Article ID 16103, 2015.

[65] T. Voigtländer, S. K. Gupta, S. Thum et al., "MicroRNAs in serum and bile of patients with primary sclerosing cholangitis and/or cholangiocarcinoma," PLoS ONE, vol. 10, no. 10, Article ID e0139305, 2015.

[66] R. Silakit, W. Loilome, P. Yongvanit et al., "Circulating miR192 in liver fluke-associated cholangiocarcinoma patients: a prospective prognostic indicator," Journal of Hepato-BiliaryPancreatic Sciences, vol. 21, no. 12, pp. 864-872, 2014.
[67] J. Plieskatt, G. Rinaldi, Y. Feng et al., "A microRNA profile associated with Opisthorchis viverrini-induced cholangiocarcinoma in tissue and plasma," BMC Cancer, vol. 15, no. 1, article no. 309, 2015.

[68] M. Kojima, H. Sudo, J. Kawauchi et al., "MicroRNA markers for the diagnosis of pancreatic and biliary-tract cancers," PLoS ONE, vol. 10, no. 2, Article ID e0118220, 2015.

[69] A. Chandra, A. Ray, S. Senapati, and R. Chatterjee, "Genetic and epigenetic basis of psoriasis pathogenesis," Molecular Immunology, vol. 64, no. 2, pp. 313-323, 2015.

[70] A. Gupta, A. Sharma, A. Yadav et al., "Evaluation of miR27a, miR-181a, and miR-570 genetic variants with gallbladder cancer susceptibility and treatment outcome in a north indian population," Molecular Diagnosis and Therapy, vol. 19, no. 5, pp. 317-327, 2015.

[71] Q. Sun, H. Gu, Y. Zeng et al., "Hsa-mir-27a genetic variant contributes to gastric cancer susceptibility through affecting miR-27a and target gene expression," Cancer Science, vol. 101, no. 10, pp. 2241-2247, 2010.

[72] J. Xu, Z. Yin, H. Shen et al., "A genetic polymorphism in premiR-27a confers clinical outcome of non-small cell lung cancer in a Chinese population," PLoS ONE, vol. 8, no. 11, Article ID e79135, 2013.

[73] Z. Wang, J. Lai, Y. Wang, W. Nie, and X. Guan, “The Hsa-miR27a rs895819 (A>G) polymorphism and cancer susceptibility," Gene, vol. 521, no. 1, pp. 87-90, 2013.

[74] Y. Lin, Y. Nie, J. Zhao et al., "Genetic polymorphism at miR181a binding site contributes to gastric cancer susceptibility," Carcinogenesis, vol. 33, no. 12, pp. 2377-2383, 2012.

[75] J.-Y. Ma, H.-J. Yan, Z.-H. Yang, and W. Gu, "Rs895819 within miR-27a might be involved in development of non small cell lung cancer in the Chinese Han population," Asian Pacific Journal of Cancer Prevention, vol. 16, no. 5, pp. 1939-1944, 2015.

[76] Z. Wang, X. Sun, Y. Wang, X. Liu, Y. Xuan, and S. Hu, "Association between miR-27a genetic variants and susceptibility to colorectal cancer," Diagnostic Pathology, vol. 9, no. 1, article no. 146, 2014.

[77] Y. Deng, H. Bai, and H. Hu, "rs11671784 G/A variation in miR27a decreases chemo-sensitivity of bladder cancer by decreasing miR-27a and increasing the target RUNX-1 expression," Biochemical and Biophysical Research Communications, vol. 458, no. 2, pp. 321-327, 2015.

[78] D. Shi, P. Li, L. Ma et al., "A genetic variant in pre-miR-27a is associated with a reduced renal cell cancer risk in a Chinese population," PLoS ONE, vol. 7, no. 10, Article ID e46566, 2012.

[79] K. Srivastava, A. Srivastava, and B. Mittal, "Common genetic variants in pre-microRNAs and risk of gallbladder cancer in North Indian population," Journal of Human Genetics, vol. 55, no. 8, pp. 495-499, 2010.

[80] F. Mihalache, A. Höblinger, M. Acalovschi, T. Sauerbruch, F. Lammert, and V. Zimmer, "A common variant in the precursor miR-146a sequence does not predispose to cholangiocarcinoma in a large european cohort," Hepatobiliary and Pancreatic Diseases International, vol. 11, no. 4, pp. 412-417, 2012.

[81] A. Shinozaki, T. Sakatani, T. Ushiku et al., "Downregulation of MicroRNA-200 in EBV-associated gastric carcinoma," Cancer Research, vol. 70, no. 11, pp. 4719-4727, 2010.

[82] M. Zhou, Z. Liu, Y. Zhao et al., "MicroRNA-125b confers the resistance of breast cancer cells to paclitaxel through suppression of pro-apoptotic Bcl-2 antagonist killer 1 (Bak1) expression," Journal of Biological Chemistry, vol. 285, no. 28, pp. 2149621507, 2010. 
[83] C.-C. Lin, W. Jiang, R. Mitra, F. Cheng, H. Yu, and Z. Zhao, "Regulation rewiring analysis reveals mutual regulation between STAT1 and miR-155-5p in tumor immunosurveillance in seven major cancers," Scientific Reports, vol. 5, Article ID 12063 , 2015.

[84] J. H. Hwang, J. Voortman, E. Giovannetti et al., "Identification of microRNA-21 as a biomarker for chemoresistance and clinical outcome following adjuvant therapy in resectable pancreatic cancer," PLoS ONE, vol. 5, no. 5, Article ID e10630, 2010. 


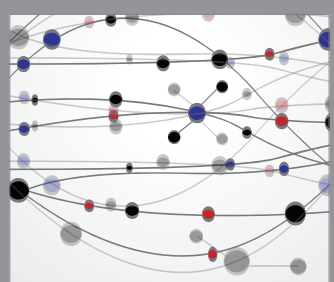

The Scientific World Journal
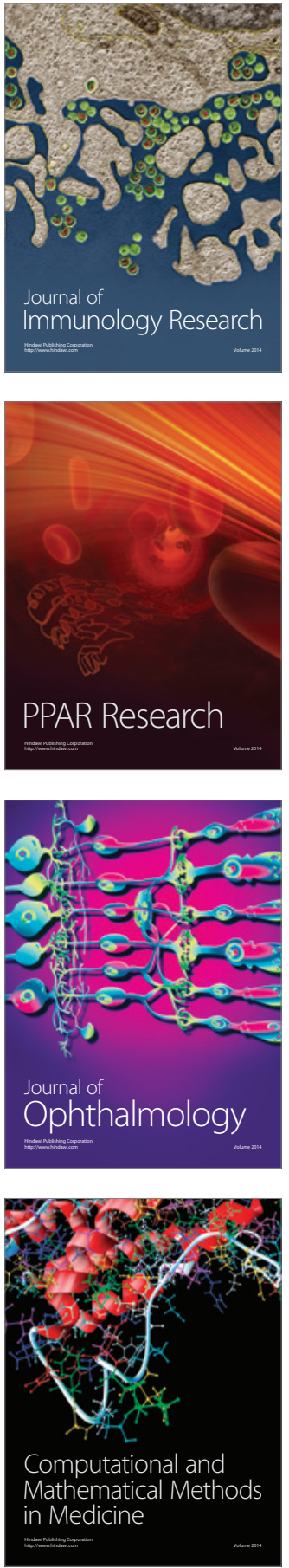

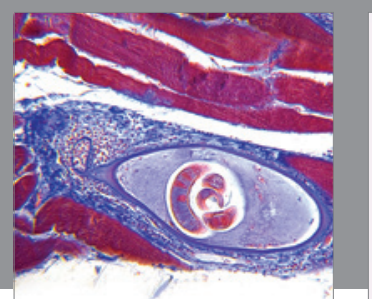

Gastroenterology Research and Practice

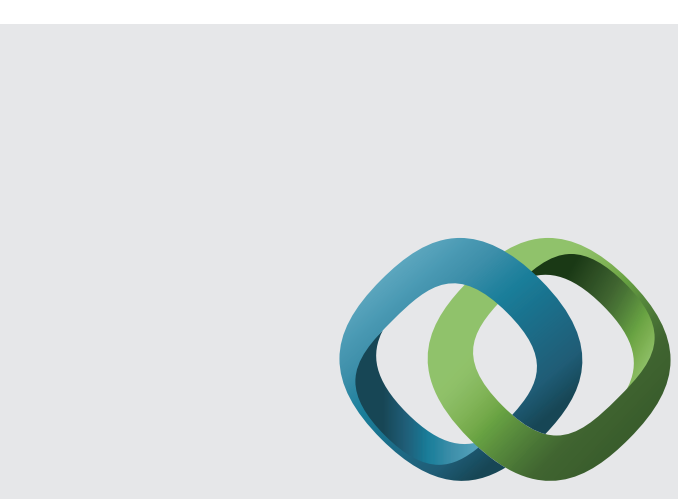

\section{Hindawi}

Submit your manuscripts at

http://www.hindawi.com
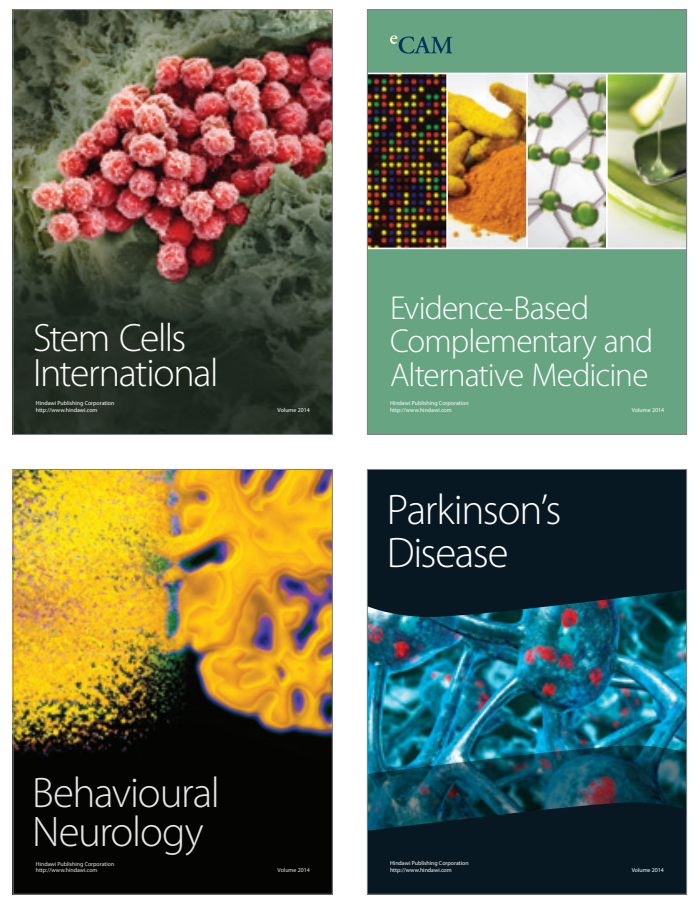
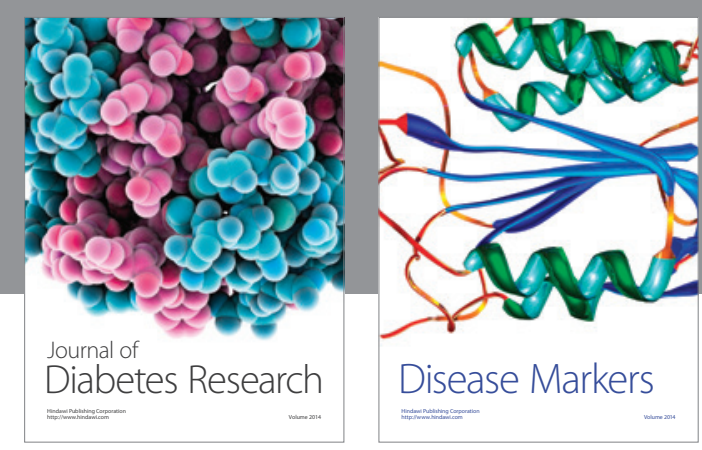

Disease Markers
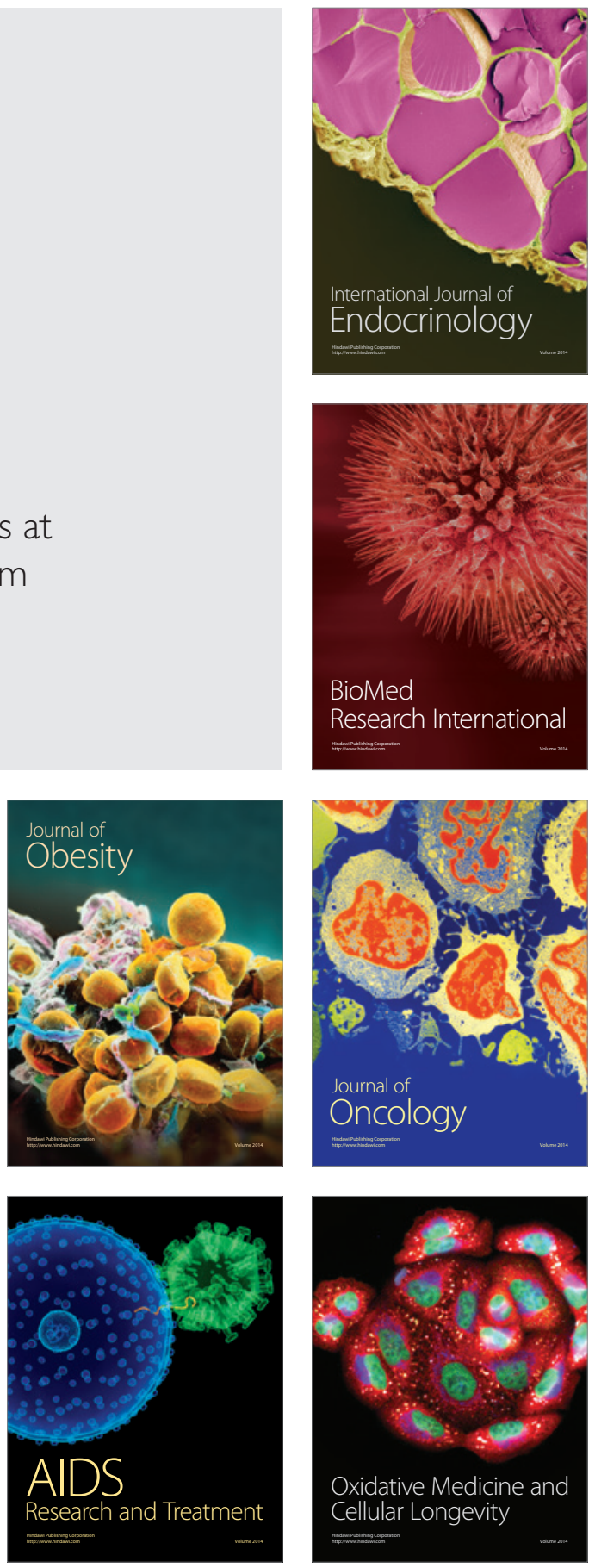